\title{
Physiological and psychological outcomes of kangaroo mother care of preterm infants-An overview
}

\begin{abstract}
Background: Neonatal care especially for premature infants may require varying periods of intensive care which may limit parental involvement. Kangaroo mother care (KMC) allows for close contact between mother and infant, widely used by Indigenous populations but now introduced into neonatal nurseries. This paper reviews the growing literature highlighting the physiological and psychological impact of its introduction in the care of preterm infants.
\end{abstract}

Methods: A brief introduction of current practices in the care of preterm infants is followed by discussing the rationale of KMC. The benefits or otherwise of such care is reviewed, drawing on the current literature. Future avenues of study are suggested.

Results: KMC has been successfully undertaken of preterm infants. Such care improved the infant's circulatory physiological parameters which included heart rate variability, oxygen saturations and temperature control. There also appeared to be a reduction in pain scores during uncomfortable neonatal procedures. An increase in successful breastfeeding, improved maternal-infant interaction and better neurodevelopmental progress has also been observed. Further study may utilise vital signs to affirm clinical outcomes. The administration of the relatively straight forward measure of the Alarm Distress Baby Scale may objectively assess infant well-being and their resultant social interactions.

Conclusion: The current literature suggests multiple benefits for preterm infants exposed to KMC with improvement in physiological parameters and developmental outcomes. It also empowers mothers to be more intimately involved with their infants. These reported findings encourage the safe introduction of $\mathrm{KMC}$ into further nurseries.

Keywords: kangaroo mother care, preterm infants, skin-to-skin contact, pain relief, breastfeeding, development
Volume 7 Issue I - 202 I

\author{
Krisha Changrani,' Samuel Menahem ${ }^{2,3}$ \\ 'Department of Medical Education, University of Melbourne, \\ Australia \\ ${ }^{2}$ Department of Paediatrics, University of Melbourne, Australia \\ ${ }^{3}$ Department of Obstetrics and Gynaecology, Monash University, \\ Australia
}

Correspondence: Samuel Menahem, Monash University, Victoria 3800, Australia, Tel 61-3-95091333, Fax 61-3-95761352, Email samuel.menahem@monash.edu

Received: November 17, 2020 | Published: January 27, 2021
Abbreviations: KMC, kangaroo mother care; NICU, neonatal intensive care unit; $\mathrm{SCN}$, special care nursery; $\mathrm{ADBB}$, alarm distress baby scale

\section{Introduction}

\section{Importance of mother-infant attachment}

Early experiences and in particular, the mother-infant relationship, have a lifelong impact on each individual. ${ }^{1}$ Preterm infants frequently require long term hospitalisation prior to their discharge. Disorganised mother-infant attachment and ongoing social relationship problems have been reported to be more prevalent amongst very preterm or very low birth weight infants. ${ }^{2}$ Of interest mothers who saw their newborn on the first day of their admission to the neonatal intensive care unit (NICU) had higher mean maternal attachment scores, compared to those who saw their baby in the subsequent days. These findings suggest that if a mother achieves early attachment and interacts with her infant shortly after delivery, a stronger sense of motherhood and a healthier relationship may eventuate. ${ }^{3}$

The terminology of kangaroo mother care (KMC) comes from the Australian kangaroo which carries her joey in her pouch located on the front of her abdomen (Figure 1). After birth, the joey weighing approximately $2 \mathrm{~g}$, crawls over the mother kangaroo's fur to arrive at her pouch in approximately 3 minutes and suckles milk from the mother's teats. Feeding and growth of the joey occurs in this safe environment for an average of 235 days, after which the joey continues to develop outside the mother's pouch. ${ }^{4}$ Whilst KMC is a relatively new approach, breastfeeding on demand and extensive carrying of an infant on the mother's body is an age-old practice. The custom of continuously carrying infants in a shawl or sling wearing no or minimal clothing was noted to be common in warmer climates, notable in native tribal groups of Central America and Siberia. Physical closeness was maintained during the night, with mothers and infants sleeping next to each other in the same bed. Despite the colder and harsher climates, Eskimo tribes have been observed practicing swaddling and using shawls to carry their infants on the mother's back. $^{5}$ !Kung San hunter-gatherers in North-Western Botswana were also known to hold or carry their infants $80-90 \%$ of the time during the infant's first few months of life. ${ }^{6}$ These practices by tribal groups were found to reduce stress responses and cortisol reactivity, and have a calming effect on their infants (Table 1). ${ }^{6}$

\section{Current care of preterm infants}

Infants born before 37 weeks gestation are considered 'preterm'.7 Those born at 34.0 to 36.6 weeks gestation may be allowed immediate 
skin-to-skin contact with their mother with attempted feeding within the first hour of life and then again three hours later. However, due to the increased risk of hypoglycaemia, hypothermia, the early onset of respiratory distress and/or apnoea, sepsis, and the development of hyperbilirubinemia, feeding difficulties, and the potential of poor weight gain, most of these infants are admitted to the NICU or special care nursery $(\mathrm{SCN})$ for observation and anticipatory management. ${ }^{8}$ Preterm infants are at higher risk of heat loss due to decreased thermal insulation from brown adipose tissue, a disproportionate ratio of body surface area to mass, laying in a posture of extension, possessing a relatively thin epidermis with reduced permeability and poorer vasomotor control. ${ }^{9}$ For this reason, preterm infants need their temperature monitored, being placed in incubators or exposed to radiant warmers, which may result in prolonged separation from their mothers (Table 2).

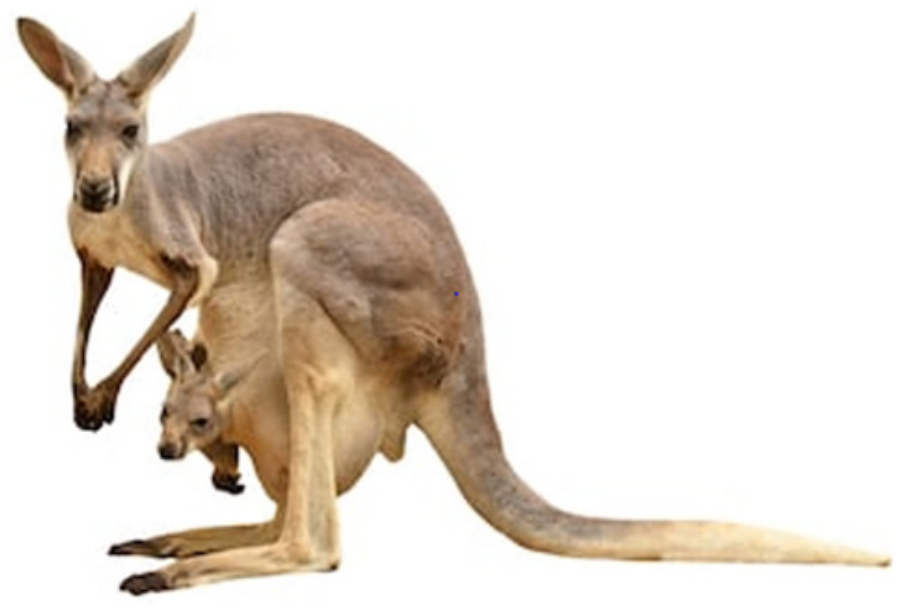

Figure I Joey in the pouch of her mother kangaroo.

Table I Summary of physiological and developmental \& psychological benefits of KMC (See Appendix)

\begin{tabular}{|c|c|}
\hline Physiological benefits & Psychological benefits \\
\hline $\begin{array}{l}\text { Increased Parasympathetic Activity }{ }^{16-18} \\
\text { Decreased Heart Rate } \\
\text { Decreased Respiratory Rate } \\
\text { Increased Oxygen Saturations }{ }^{14} \\
\text { Increased Temperature }{ }^{14} \\
\text { Increased Cerebral Blood Flow }{ }^{18,19} \\
\text { Decreased Secondary Infections }{ }^{20} \\
\text { Decreased Salivary Oxytocin Concentrations }{ }^{22,23} \\
\text { Pain Relief for Painful Procedures }{ }^{27-37} \\
\text { Improved Weight Gains }{ }^{14,20,38,45} \\
\text { Greater Success in Establishing Early Breastfeeding }{ }^{39-45}\end{array}$ & $\begin{array}{l}\text { Improved Early Cognitive Performance }{ }^{47} \\
\text { Improved Communication Performance }{ }^{47} \\
\text { Improved Neurobehavioural Performance }{ }^{14,48} \\
\text { Increased Alertness and Decreased Gaze Aversion }{ }^{48} \\
\text { Increased Mother-Infant Interaction }{ }^{48,49} \\
\text { Improved Maternal Affect }{ }^{48} \\
\text { Improved Maternal Response to Infant Cues }{ }^{48}\end{array}$ \\
\hline
\end{tabular}

Table 2 Difficulties in the introduction of KMC

Under-resourced environments
Lack of suitable facilities
Poor nursing support or training
Fear and anxiety of hurting the infant
Limited clinical staff
Separation of infant needing interventions
Cultural issues in handling of newborn infants
Limited awareness of KMC

It is estimated that there are over 15 million preterm births worldwide each year, with the great majority in developing countries. ${ }^{10}$ Neonatal care of premature infants is complex. It requires expensive infrastructure and trained personnel. In many countries with limited resources NICUs and SCNs are often understaffed and/or illequipped. ${ }^{11}$ To address some of these issues KMC was first proposed in 1978 by Edgar Rey ${ }^{12}$ as an adjunct to conventional neonatal care.

\section{Kangaroo mother care}

$\mathrm{KMC}$ is the practice of early, continuous and prolonged skin-to-skin contact between a mother and her newborn, exclusive breastfeeding whenever possible with the aim for an earlier discharge of low birth weight and or preterm infants. The baby is held upright between the mother's breasts wearing only a diaper and woollen cap, supported by a cloth wrapping around the naked torso of the mother and baby (Figure 2) to maintain ongoing contact whenever possible between mother and infant. Separation only occurs to attend to mothers' personal needs or for interventions to the infant. If the mother and newborn are well, KMC is recommended immediately after birth once the umbilical cord is cut and the infant dried and examined. ${ }^{10}$ Preterm infants requiring respiratory support via continuous positive airway pressure or endotracheal intubation, require a skilled nurse to be present at all times with regular assessments of the infant. ${ }^{13}$ $\mathrm{KMC}$ can be performed continuously or intermittently, such as for 120 minutes daily or for 15 minutes before or during invasive procedures. ${ }^{14}$ Following the introduction of $\mathrm{KMC}$, more than 500 low birth weight infants in Bogota, Columbia were exposed to KMC, which was associated with a tripling in infant survival rates. ${ }^{15}$ What 
then are the reported physiological and psychological outcomes of KMC of preterm infants? To answer this question an extensive literature review was undertaken of studies reported in the English language using the Preferred Reporting Items for Systematic Reviews and Meta-Analyses (PRISMA) reporting guidelines.

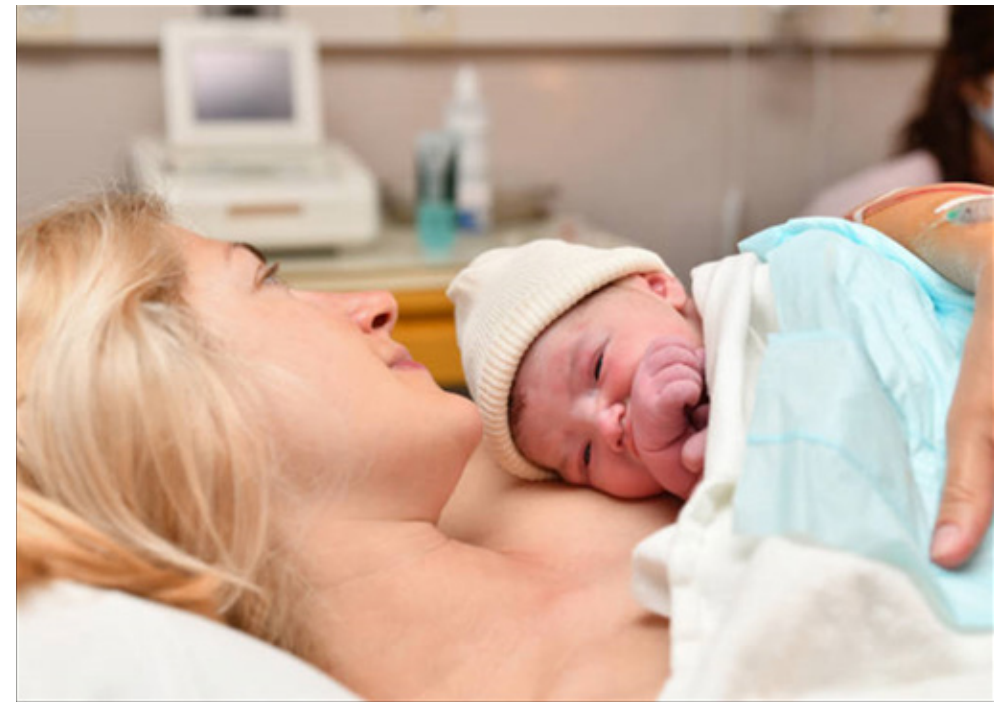

Figure 2 Skin-to-skin contact between mother and infant.

Appendix I Summary of published findings

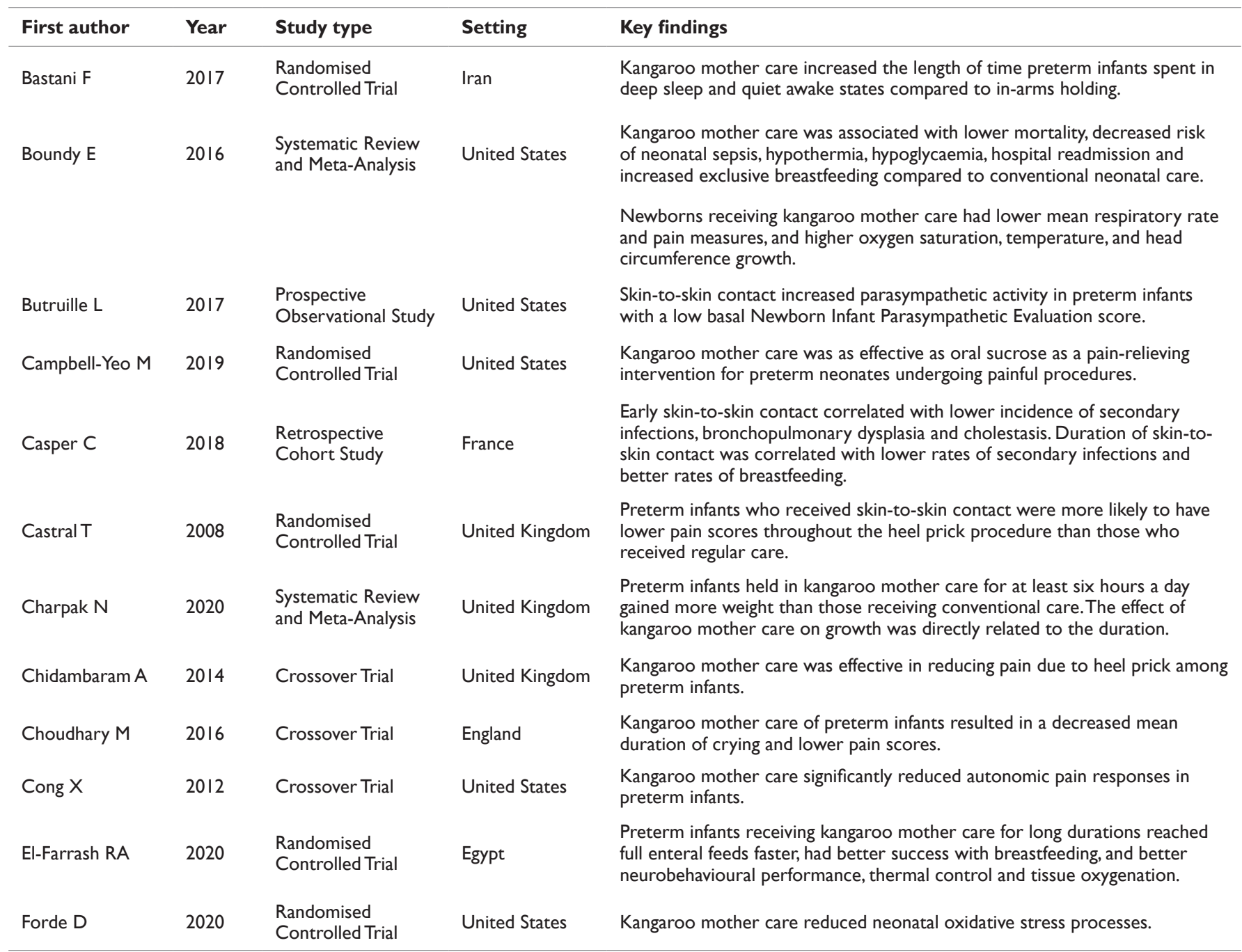


Table Continued...

\begin{tabular}{|c|c|c|c|c|}
\hline First author & Year & Study type & Setting & Key findings \\
\hline Gonya J & 2017 & $\begin{array}{l}\text { Retrospective } \\
\text { Cohort Study }\end{array}$ & United States & $\begin{array}{l}\text { Early and frequent skin-to-skin care of extremely preterm infants were } \\
\text { associated with early cognitive and communication performance. }\end{array}$ \\
\hline Head L & 2014 & Systematic Review & United States & $\begin{array}{l}\text { Short-term benefits of kangaroo mother care on preterm infants included } \\
\text { improved neurodevelopment. }\end{array}$ \\
\hline Johnston C & 2017 & Systematic Review & United States & $\begin{array}{l}\text { Skin-to-skin care was effective, as measured by composite pain indicators, and } \\
\text { safe for a single painful procedure. }\end{array}$ \\
\hline Karimi S & 2020 & $\begin{array}{l}\text { Randomised } \\
\text { Controlled Trial }\end{array}$ & Iran & $\begin{array}{l}\text { Kangaroo mother care improved neonatal weight gain, breastfeeding and } \\
\text { decreased the duration of hospitalisation of preterm infants. }\end{array}$ \\
\hline Kashaninia Z & 2015 & $\begin{array}{l}\text { Randomised } \\
\text { Controlled Trial }\end{array}$ & Iran & $\begin{array}{l}\text { Premature neonates who received kangaroo mother care had greater weight } \\
\text { gain than those that did not. }\end{array}$ \\
\hline Kommers D & 2018 & $\begin{array}{l}\text { Prospective } \\
\text { Observational Study }\end{array}$ & Netherlands & $\begin{array}{l}\text { The salivary oxytocin concentration in the pooled saliva of preterm infant } \\
\text { twins decreased during kangaroo mother care. }\end{array}$ \\
\hline Korraa A & 2014 & $\begin{array}{l}\text { Prospective } \\
\text { Observational Study }\end{array}$ & United Kingdom & $\begin{array}{l}\text { Kangaroo mother care improved cerebral blood flow in preterm and low } \\
\text { birth weight infants. }\end{array}$ \\
\hline Lyngstad L & 2014 & Crossover Trial & Ireland & $\begin{array}{l}\text { Skin-to-skin contact of premature infants resulted in significantly lower stress } \\
\text { levels for diaper change compared to those cared for in an incubator/bed for } \\
\text { preterm infants. }\end{array}$ \\
\hline Mekonnen A & 2019 & Meta-Analysis & United Kingdom & $\begin{array}{l}\text { Preterm and low birth weight infants receiving kangaroo mother care } \\
\text { initiated breastfeeding } 2 \text { days } 14 \text { hours and } 24 \text { minutes earlier than } \\
\text { conventional care of radiant warmer/incubator method. }\end{array}$ \\
\hline Moore ER & 2016 & Systematic Review & United Kingdom & Skin-to-skin contact can be used to promote breastfeeding. \\
\hline Morelius E & 2015 & $\begin{array}{l}\text { Randomised } \\
\text { Controlled Trial }\end{array}$ & Ireland & $\begin{array}{l}\text { Preterm infants receiving almost continuous skin-to-skin care had a } \\
\text { decreased cortisol reactivity in response to handling, had improved } \\
\text { concordance between mothers' and infants' salivary cortisol levels. }\end{array}$ \\
\hline Mosayebi Z & 2014 & $\begin{array}{l}\text { Randomised } \\
\text { Controlled Trial }\end{array}$ & Iran & $\begin{array}{l}\text { Kangaroo mother care of preterm infants resulted in lower pain intensity } \\
\text { scores during the heel-lancing procedure. }\end{array}$ \\
\hline Mwendwa A & 2012 & $\begin{array}{l}\text { Randomised } \\
\text { Controlled Trial }\end{array}$ & Kenya & $\begin{array}{l}\text { Low birth weight infants received partial kangaroo mother care grew faster } \\
\text { and were discharged earlier than those receiving standard care. }\end{array}$ \\
\hline Nimbalkar S & 2013 & Crossover Trial & India & $\begin{array}{l}\text { Short duration kangaroo mother care ( } 15 \text { minutes) had stress reducing } \\
\text { benefits. Preterm infants above } 32 \text { weeks' gestational age receiving kangaroo } \\
\text { mother care benefited from decreased pain during the heel prick procedure. }\end{array}$ \\
\hline Nimbalkar S & 2020 & Crossover Trial & India & $\begin{array}{l}\text { Skin-to-skin care and sucrose had comparable clinical efficacy for preterm } \\
\text { neonatal pain control. }\end{array}$ \\
\hline Oras $\mathrm{P}$ & 2016 & $\begin{array}{l}\text { Prospective } \\
\text { Observational Study }\end{array}$ & Sweden & $\begin{array}{l}\text { A longer daily duration of skin-to-skin contact of preterm infants in the } \\
\text { neonatal intensive care unit was associated with earlier attainment of } \\
\text { breastfeeding. }\end{array}$ \\
\hline Parsa P & 2018 & $\begin{array}{l}\text { Randomised } \\
\text { Controlled Trial }\end{array}$ & Iran & $\begin{array}{l}\text { Kangaroo mother care of premature infants enhanced physiological } \\
\text { indices: heart rate, respiratory rate, arterial blood oxygen saturation and } \\
\text { temperature. }\end{array}$ \\
\hline Samra N & 2012 & $\begin{array}{l}\text { Prospective } \\
\text { Observational Study }\end{array}$ & Egypt & $\begin{array}{l}\text { Neonates who received kangaroo mother care recovered earlier from } \\
\text { jaundice and required a shorter duration of phototherapy than the control } \\
\text { group. }\end{array}$ \\
\hline Sehgal A & 2020 & $\begin{array}{l}\text { Prospective } \\
\text { Observational Study }\end{array}$ & Australia & $\begin{array}{l}\text { Kangaroo mother care resulted in significant circulatory benefits in preterm } \\
\text { infants. }\end{array}$ \\
\hline Sen $\mathrm{E}$ & 2020 & $\begin{array}{l}\text { Randomised } \\
\text { Controlled Trial }\end{array}$ & United States & $\begin{array}{l}\text { Kangaroo mother care was more effective than oral sucrose in pain relief } \\
\text { during heel-lancing in preterm infants. }\end{array}$ \\
\hline Sharma D & 2018 & $\begin{array}{l}\text { Randomised } \\
\text { Controlled Trial }\end{array}$ & India & $\begin{array}{l}\text { Early shifting of preterm infants to kangaroo care ward with birth weight of } \\
1000 \text { grams to I I00 grams led to better growth and was cost effective. }\end{array}$ \\
\hline Sharma D & 2016 & $\begin{array}{l}\text { Randomised } \\
\text { Controlled Trial }\end{array}$ & India & $\begin{array}{l}\text { Early baby care in kangaroo care ward was equally efficacious as baby care } \\
\text { in intermediate intensive care in improving the growth outcomes of stable } \\
\text { preterm infants reaching term gestational age. }\end{array}$ \\
\hline
\end{tabular}


Table Continued...

\begin{tabular}{lllll}
\hline First author & Year & Study type & Setting & Key findings \\
Shattnawi K & 2019 & $\begin{array}{l}\text { Randomised } \\
\text { Controlled Trial }\end{array}$ & United States & $\begin{array}{l}\text { Premature infants who received skin-to-skin contact demonstrated higher } \\
\text { weight gain from day 3-5, experienced significantly fewer episodes of apnoea } \\
\text { and were less likely to use formula feeding at discharge. }\end{array}$ \\
Shukla V & 2017 & $\begin{array}{l}\text { Randomised } \\
\text { Controlled Trial }\end{array}$ & Netherlands & $\begin{array}{l}\text { The pain reducing potential of sucrose and kangaroo mother care were } \\
\text { comparable. }\end{array}$ \\
Zhang F & 2012 & Review Article & United Kingdom & $\begin{array}{l}\text { Kangaroo mother care improved the nurturing of premature infants and } \\
\text { reduced the risk factors of oral defects. }\end{array}$
\end{tabular}

\section{Results}

The search identified 173 articles in Medline (Ovid) and 277 articles in Embase (Ovid). After removal of 94 duplicate studies, 356 records remained, of which 306 were excluded following review of their abstracts. Exclusion criteria included limited clinical reports, oral and poster presentations, concerns about study design and an inability to obtain full text access. This review reports the findings of the remaining 39 full text articles.

\section{Physiological outcomes}

\section{Cardiovascular and metabolic indices}

$\mathrm{KMC}$ of preterm infants was noted to enhance physiological indices. Butruille et al., ${ }^{16}$ conducted a study analysing parasympathetic activity of mothers and preterm infants using heart rate variability analysis. They noted an activation of parasympathetic activity with skin-to-skin contact. In a quasi-experimental study of 100 preterm infants, Parsa et al., ${ }^{17}$ observed a significant decrease in heart rate and respiratory rate, and an increase in arterial blood oxygen saturation and temperature in infants who received KMC compared to standard neonatal care. A prospective study by Sehgal et al., ${ }^{18}$ of 40 stable preterm infants conducted at a quaternary centre assessed the impact of skin-to-skin parent-infant care on preterm circulatory physiology. They documented significant physiological benefits on cardiac function which included a non-significant increase in axillary temperature, a significant increase in cardiac contractility and tricuspid annular plane systolic excursion, decreased measures of pulmonary vascular resistance and an increase in systemic cardiac output. That was associated with increased cerebral blood flow and reduced middle cerebral artery resistive index. These circulatory benefits were also noted by El-Farrash et al., ${ }^{14}$ and Korraa et al. ${ }^{19}$

Additional benefits of KMC included a lower incidence of secondary infections, bronchopulmonary dysplasia and cholestasis following early commencement of skin-to-skin contact. ${ }^{20}$ Furthermore there was an increased length of time spent in deep sleep and quiet awake states, ${ }^{21}$ decreased salivary oxytocin concentrations ${ }^{22}$ and improved salivary cortisol concordance between mother and infant. ${ }^{23}$ There was a reduction in the duration of phototherapy required for the treatment of jaundice despite the KMC being intermittent. ${ }^{24}$ Studies have also reported the effect of KMC on neonatal oxidative stress processes in preterm infants. Forde et al. ${ }^{25}$ utilised urinary allantoin as a biomarker for oxidative stress. They noted significant reductions in mean allantoin levels in preterm infants subjected to KMC compared to those in standard neonatal care.

\section{Pain relief}

The value of KMC in alleviating pain experienced by preterm infants has been widely studied. Preterm infants often spend weeks in the NICU. They undergo approximately fourteen daily painful procedures. Only one-third receive appropriate analgesic therapy. Painful procedures include blood sampling by routine heel pricks, suctioning of the oropharynx and airway, immunisations, changes in dressings and surgical procedures. ${ }^{26}$ Campbell-Yeo et al. ${ }^{27}$ conducted a randomised controlled trial involving 242 stable preterm infants. They concluded that KMC was equally effective as $24 \%$ oral sucrose at reducing behavioural pain intensity scores associated with painful procedures. There was evidence that KMC has a sustained efficacy over repeated procedures, and the combination of KMC and sucrose did not provide additional benefit. Castral et al., ${ }^{28}$ conducted a randomised controlled trial of 59 stable preterm infants, comparing those who received 15 minutes of skin-to-skin contact with mother before, during and following a heel prick, compared to those receiving standard care. Preterm infants in the former group were observed to have lower neonatal facial coding system scores compared to the control group. In addition, Choudhary et al. ${ }^{29}$ noted a reduction in the mean duration of crying in preterm neonates who received KMC. Cong et al., ${ }^{30}$ concluded KMC before and during heel stick procedures, stabilised the infant's heart rate, whilst longer skin-to-skin contact of 30 minutes significantly improved their autonomic pain responses compared to a shorter period of 15 minutes or being in an isolate. These findings were supported by further randomised controlled trials conducted by Chidambaram et al., ${ }^{31}$ Mosayebi et al., ${ }^{32} \&$ Sen et al. ${ }^{33}$ In two studies by Nimbalkar et al., ${ }^{34,35}$ skin-to-skin contact and oral sucrose were noted to have comparable clinical efficacy. Benefit was also noted even with short periods of KMC (15 minutes). Shukla et al., ${ }^{36}$ similarly concluded that there was no difference in pain reducing effects of sucrose compared to KMC. Lyngstad et al., ${ }^{37}$ additionally noted that skin-to-skin contact reduced the stress levels of preterm infants during diaper changes, compared to those that remained in an incubator or cot, as experienced by changes in the Skin Conductance Algesimeter. KMC appears to be effective in reducing pain of preterm infants with effects noted to be equal or superior to oral sucrose. While there is the ease of administration of oral sucrose, KMC may be preferable for in addition to the pain relief, there are additional beneficial effects.

\section{Achievement of breastfeeding and weight gains}

$\mathrm{KMC}$ of preterm infants appear to improve their weight gains in addition to facilitating early breastfeeding. Casper et al., ${ }^{20}$ conducted a retrospective study of 26 premature infants receiving skin-to-skin contact in the NICU and SCN. There was a statistically significant increase in the rate of breastfeeding at discharge in infants who commenced skin-to-skin contact in their 1st week of life, compared to those who started a week later. The frequency of skin-to-skin contact improved the duration of breastfeeding prior to discharge, but was not statistically significant. In the randomised controlled trial conducted by El-Farrash et al., ${ }^{14}$ preterm infants who received KMC scored 
significantly higher on an Infant Breastfeeding Assessment Tool and reached full enteral feeding earlier as compared to controls. The best breastfeeding improvement was noted in infants who received KMC for 120 minutes daily. A randomised controlled trial of 104 preterm infants conducted by Oras et al. ${ }^{38}$ similarly noted that longer duration of KMC in the NICU was associated with earlier attainment of exclusive breastfeeding. Improvements in the baby's weight gain with KMC were also noted in studies conducted by Karimi et al., ${ }^{39}$ Kashaninia et al., ${ }^{40}$ Mwendwa et al., ${ }^{41}$ Sharma et al.,44,43 and Shattnawi et $\mathrm{al}^{44}$ In a meta-analysis by Mekonnen et al. ${ }^{45}$ the aggregate mean time to initiate breastfeeding among preterm and low birth weight infants receiving $\mathrm{KMC}$ was 2.6 days, a full 2 days, 14 hours and 24 minutes earlier than those still being cared for in incubators or exposed to radiant warmers. The current literature suggests that early $\mathrm{KMC}$ of preterm infants resulted in improved breastfeeding success and weight gains compared to conventional neonatal care. Further studies are required to determine the optimal duration of KMC to achieve a positive response.

\section{Developmental and psychological outcomes}

Preterm infants born before 30 weeks' gestation may have poorer neurodevelopmental prognoses ${ }^{46}$ Results from a retrospective cohort study by Gonya et al., ${ }^{47}$ of extremely preterm infants admitted to a NICU suggested an association between early and frequent skin-toskin contact and early cognitive and communication performance. Preterm infants who received KMC from either their mother or father, for longer periods with an increased frequency or greater total hours of contact, performed better. El-Farrash et al., ${ }^{14}$ documented smoother movements, symmetrical responses, decreased handling requirements, and less excitability and reduced periods of lethargy amongst preterm infants who received KMC daily for at least 7 days consecutively, compared to those who received conventional neonatal care. This was partially explained by the theoretical multimodal sensory stimulation that $\mathrm{KMC}$ is thought to provide, accelerating neurological maturation.

The separation of mothers from their preterm infants following their admission to the NICU and the clinical interventions required, may delay opportunities for mother-infant attachment. Feldman et al., ${ }^{48}$ compared the impact of KMC and traditional care on parenting outcomes and the impact on preterm infant development. The study included 73 preterm infants who received KMC in the NICU and 73 control infants who received standard incubator care, matched for birth weight, gestational age, medical severity and demographics. The study noted that KMC positively influenced parental perceptions and behaviours, and infant development independent of the infant's medical status. Following KMC, mothers displayed a more positive affect, with improved handling of their infant being better attuned to their infant cues. The infants were more alert with less gaze aversion. The study speculated that KMC had a direct impact on infant development through improved neurophysiological organisation and indirectly though improving parental mood, perceptions and interactive behaviour. An earlier study by Ahn et al., ${ }^{49}$ also concluded that maternal attachment scores were higher among mothers involved in $\mathrm{KMC}$, compared to the conventional neonatal practices in a cohort of Korean premature infants and their mothers.

Sahlén et al., ${ }^{50}$ conducted a randomised controlled trial which compared the effects of continuous versus intermittent skin-to-skin contact from birth to discharge in premature infants on mother-infant interaction. The results of this study however did not support the hypothesis that continuous skin-to-skin contact improved motherinfant interaction - there were no statistically significant differences in mother-infant interaction between the two groups. The researchers proposed that while skin-to-skin contact creates a foundation for greater contact with the infants and increased interaction, there might be a need for more specific interventions to enhance such interactions between the mother and infant. Limitations of that study included breastfeeding as a possible confounder, as it fostered skin-to-skin contact and has been previously described to positively improve the interactions.

\section{Further study}

The introduction of KMC is limited by the availability of suitable hospital settings that ensure comfort and privacy for mothers and their infants. The variety of those settings still need be determined. The observed improvements in physiological parameters arising from $\mathrm{KMC}$ may be more readily documented by analysing routinely collected vital signs of stable preterm infants, including changes in heart and breathing rates and oxygen saturations. The results may further facilitate wider adoption of KMC as the "standard care" of preterm infants.

The ability of an infant to engage with caregivers, through initiating and maintaining eye contact, vocalising, and using facial expression and body movements, emerge during the first two months of life. ${ }^{51,52}$ During periods of severe stress for an infant, either due to organic disease or adverse psychological factors, a pattern of social withdrawal and inactivity can be observed. This reaction supports survival through a conservation of resources, until the perceived threat or distressing circumstances become more favourable. This phenomenon is described as conservation-withdrawal. ${ }^{53,54}$ The Alarm Distress Baby Scale (ADBB), an 8-item assessment tool may be used to objectively evaluate social withdrawal of infants between 2 and 24 months. ${ }^{55}$ A recent review on the use of the ADBB in assessing social withdrawal in infants with an underlying organic illness, found the ADBB to be very helpful in assessing infant well-being with serial measurements being able to determine subsequent improvement. ${ }^{56}$ Infants who have experienced KMC may be further studied by carrying out an ADBB assessment to learn of its impact on their psychosocial development.

\section{Concerns about KMC}

Despite the reported benefits of KMC, there are several barriers which limit its integration into routine neonatal care of preterm infants. In a systematic review conducted by Seidman et al., ${ }^{57} 4$ of the top 5 barriers to KMC were resource-related. It encompassed "issues with facility environment/resources". That included staff shortages or limited space to carry out KMC, "negative impressions of staff attitudes or interactions", "fear/anxiety of hurting the infant" or dislodging a venous cannula or endotracheal tube. The "lack of help with $[\mathrm{KMC}]$ practice and other obligations", including cultural customs, were also considered limiting factors to its introduction. There was also a "low awareness of [KMC] on infant health". For countries with sparse resources, fatigue of the attending clinical staff working long hours was noted to be additional barriers to adoption of KMC.

\section{Limitations of this analysis}

The non-English literature was not extensively studied. Published studies contrasting the effect of kangaroo care provided by mothers versus fathers or surrogates of preterm infants were not reviewed. Furthermore, as KMC is a relatively new approach there appears to be a preponderance of positive outcomes reported in the literature possibly arising from an evangelical fervour by its proponents. Limited information is available on the potential negative consequences. 


\section{Conclusion}

Kangaroo mother care of preterm infants has many reported physiological, developmental and psychological benefits. It tends to improve circulatory physiological parameters, facilitate pain relief comparable or better to that achieved by sucrose, increase the rates of early attainment of breastfeeding, and accelerates neonatal weight gain. It tends to foster mother-infant interactions and improves neurodevelopmental outcomes. There are no clear guidelines concerning the optimal duration of $\mathrm{KMC}$, with some studies reporting greater benefits with its earlier commencement and increased daily duration. However, there remain several barriers that prevent the universal uptake of kangaroo mother care in NICUs or SCNs. Further use of routine measures of vital signs may allow for ease of analysis. The ADBB may be able to provide additional information with respect to infant well-being and social interaction arising from KMC.

To support the implementation of KMC and address potential barriers there need be further education of healthcare workers, parents and families about its application, its potential benefits, as well as the expansion of resources to deliver such care possibly routinely for preterm infants. There also is the need for additional resources and the development of suitable supportive environments. Such additional resources and training may be well compensated by the improved wellbeing of the infant, allowing for earlier discharge into the care of a more confident mother.

\section{Acknowledgments}

None.

\section{Conflicts of interest}

Authors declare that there is no conflict of interest.

\section{Funding}

None.

\section{References}

1. Shonkoff JP. Capitalizing on advances in science to reduce the health consequences of early childhood adversity. JAMA Pediatr. 2016;170(10):1003-1007.

2. Wolke D, Eryigit-Madzwamuse S, Gutbrod T. Very preterm/very low birthweight infants' attachment: infant and maternal characteristics. Arch Dis Child Fetal Neonatal Ed. 2014;99(1):F70-75.

3. Kurt F, Kucukoglu S, Ozdemir A, et al. The effect of kangaroo care on maternal attachment in preterm infants. Niger J Clin Pract. 2020;23(1):2632

4. Greydanus DE, Merrick J. Newborn care: what we can learn from the kangaroo mother. Front Public Health. 2014;2:96.

5. Schön RA, Silvén M. Natural parenting - back to basics in infant care. Evol Psychol. 2007;5(1):102-183.

6. Konner M, Worthman C. Nursing frequency, gonadal function, and birth spacing among !Kung hunter-gatherers. Science. 1980;207(4432):788.

7. LaHood A, Bryant CA. Outpatient care of the premature infant. Am Fam Physician. 2007;76(8):1159-1164.

8. The Late Preterm Infant - Care and Management [Internet]. Victoria: Victorian Agency for Health Information. 2020.

9. Assisted Thermoregulation [Internet]. Melbourne: The Royal Children's Hospital Melbourne; 2020.

10. Kangaroo Mother Care: A Practical Guide [Internet]. 2003.
11. Born Too Soon: The Global Action Report on Preterm Birth [Internet]. The Partnership For Maternal, Newborn And Child Health; 2020.

12. Rey ES MH. Rational management of the premature child. Fetal Medicine Course. 1983:23-25.

13. Lorenz L, Dawson JA, Jones H, et al. Skin-to-skin care in preterm infants receiving respiratory support does not lead to physiological instability. Arch Dis Child Fetal Neonatal Ed. 2017;102(4):F339-F44.

14. El-Farrash RA, Shinkar DM, Ragab DA, et al. Longer duration of kangaroo care improves neurobehavioral performance and feeding in preterm infants: a randomized controlled trial. Pediatr Res. 2020;87(4):683-688.

15. Kangaroo Mother Care. 2020.

16. Butruille L, Blouin A, De Jonckheere J, et al. Impact of skin-to-skin contact on the autonomic nervous system in the preterm infant and his mother. Infant Behav Dev. 2017;49:83-86.

17. Parsa P, Karimi S, Basiri B, et al. The effect of kangaroo mother care on physiological parameters of premature infants in Hamadan City, Iran. Pan Afr Med J. 2018;30:89.

18. Sehgal A, Nitzan I, Jayawickreme N, et al. Impact of skin-to-skin parentinfant care on preterm circulatory physiology. J Pediatr. 2020;222:91-97.

19. Korraa AA, El Nagger AAI, Mohamed RAE-S, et al. Impact of kangaroo mother care on cerebral blood flow of preterm infants. Ital J Pediatr. 2014;40:83.

20. Casper C, Sarapuk I, Pavlyshyn H. Regular and prolonged skin-to-skin contact improves short-term outcomes for very preterm infants: a dosedependent intervention. Arch Pediatr. 2018;25(8):469-475.

21. Bastani F, Rajai N, Farsi Z, et al. The effects of kangaroo care on the sleep and wake states of preterm infants. J Nurs Res. 2017;25(3):231-239.

22. Kommers D, Broeren M, Oei G, et al. Oxytocin levels in the saliva of preterm infant twins during kangaroo care. Biol Psychol. 2018;137:18-23.

23. Morelius E, Ortenstrand A, Theodorsson E, et al. A randomised trial of continuous skin-to-skin contact after preterm birth and the effects on salivary cortisol, parental stress, depression, and breastfeeding. Early Hum Dev. 2015;91(1):63-70.

24. Samra NM, El Taweel A, Cadwell K. The effect of kangaroo mother care on the duration of phototherapy of infants re-admitted for neonatal jaundice. J Matern-Fetal Neonatal Med. 2012;25(8):1354-1357.

25. Forde D, Deming DD, Tan JC, et al. Oxidative stress biomarker decreased in preterm neonates treated with kangaroo mother care. Biol Res Nurs. 2020;22(2):188-196.

26. Williams MD, Lascelles BDX. Early neonatal pain - a review of clinical and experimental implications on painful conditions later in life. Front Pediatr. 2020;8:30.

27. Campbell-Yeo M, Johnston CC, Benoit B, Disher T, Caddell K, Vincer $M$, et al. Sustained efficacy of kangaroo care for repeated painful procedures over neonatal intensive care unit hospitalization: a singleblind randomized controlled trial. Pain. 2019;160(11):2580-2588.

28. Castral TC, Warnock F, Leite AM, et al. The effects of skin-to-skin contact during acute pain in preterm newborns. Eur J Pain. 2008;12(4):464-471.

29. Choudhary M, Dogiyal H, Sharma D, et al. To study the effect of kangaroo mother care on pain response in preterm neonates and to determine the behavioral and physiological responses to painful stimuli in preterm neonates: a study from western Rajasthan. J Matern-Fetal Neonatal Med. 2016;29(5):826-831.

30. Cong X, Cusson RM, Walsh S, et al. Effects of skin-to-skin contact on autonomic pain responses in preterm infants. J Pain. 2012;13(7):636-645.

31. Chidambaram AG, Manjula S, Adhisivam B, et al. Effect of kangaroo mother care in reducing pain due to heel prick among preterm neonates: a crossover trial. J Matern-Fetal Neonatal Med. 2014;27(5):488-490. 
32. Mosayebi Z, Javidpour M, Rahmati M, et al. The effect of kangaroo mother care on pain from heel lance in preterm newborns admitted to neonatal intensive care unit: a crossover randomized clinical trial. $J$ Compr Ped. 2014;5(4):e22214.

33. Sen E, Manav G. Effect of kangaroo care and oral sucrose on pain in premature infants: a randomized controlled trial. Pain Manag Nurs. 2020

34. Nimbalkar SM, Chaudhary NS, Gadhavi KV, et al. Kangaroo mother care in reducing pain in preterm neonates on heel prick. Indian J Pediatr. 2013;80(1):6-10

35. Nimbalkar S, Shukla VV, Chauhan V, et al. Blinded randomized crossove trial: skin-to-skin care vs. sucrose for preterm neonatal pain. J Perinatol. 2020;40(6):896-901.

36. Shukla V, Chapla A, Uperiya J, et al. Randomized control trial to compare kangaroo mother care with oral sucrose for pain management in premature neonates on heel prick. Eur J Pediatr. 2017;176(11):1520-1521.

37. Lyngstad LT, Tandberg BS, Storm H, et al. Does skin-to-skin contact reduce stress during diaper change in preterm infants? Early Hum Dev. 2014;90(4):169-172.

38. Oras P, Thernstrom Blomqvist Y, Hedberg Nyqvist K, et al. Skin-to-skin contact is associated with earlier breastfeeding attainment in preterm infants. Acta Paediatr. 2016;105(7):783-789.

39. Karimi S, Parsa P, Basiri B, et al. The effect of kangaroo mother care on nutritional status and duration of hospitalization of premature infants in Iran. J Postgrad Medical Inst. 2020;34(1):16-21.

40. Kashaninia Z, Dehghan M. The effect of kangaroo care on weight gain of premature neonates in hospitalized in neonatal intensive care units. Biosci Biotech Res. 2015;12(2):1405-1410.

41. Mwendwa AC, Musoke RN, Wamalwa DC. Impact of partial kangaroo mother care on growth rates and duration of hospital stay of low birth weight infants at the Kenyatta National Hospital, Nairobi. East Afr Med J. 2012;89(2):53-58.

42. Sharma D, Murki S, Oleti TP. Study comparing "kangaroo ward care" with "intermediate intensive care" for improving the growth outcome and cost effectiveness: randomized control trial. J Matern-Fetal Neonatal Med. 2018;31(22):2986-2993.

43. Sharma D, Murki S, Pratap OT. The effect of kangaroo ward care in comparison with "intermediate intensive care" on the growth velocity in preterm infant with birth weight $<1100 \mathrm{~g}$ : randomized control trial. Eur $J$ Pediatr. 2016;175(10):1317-1324.

44. Shattnawi KK, Al-Ali N. The effect of short duration skin to skin contact on premature infants' physiological and behavioral outcomes: a quasiexperimental study. J Pediatr Nurs. 2019;46:e24-e8.
45. Mekonnen AG, Yehualashet SS, Bayleyegn AD. The effects of kangaroo mother care on the time to breastfeeding initiation among preterm and LBW infants: a meta-analysis of published studies. Int Breastfeed J. 2019;14:12.

46. Pickler RH, McGrath JM, Reyna BA, et al. A model of neurodevelopmental risk and protection for preterm infants. J Perinat Neonatal Nurs. 2010;24(4):356-365.

47. Gonya J, Ray WC, Rumpf RW, et al. Investigating skin-to-skin care patterns with extremely preterm infants in the NICU and their effect on early cognitive and communication performance: A retrospective cohort study. BMJ Open. 2017;7(3):e012985.

48. Feldman R, Eidelman AI, Sirota L, et al. Comparison of skin-to-skin (kangaroo) and traditional care: parenting outcomes and preterm infant development. Pediatrics. 2002;110(1 Pt 1):16-26.

49. Ahn HY, Lee J, Shin HJ. Kangaroo care on premature infant growth and maternal attachment and post-partum depression in South Korea. $J$ Trop Pediatr. 2010;56(5):342-344.

50. Sahlén Helmer C, Birberg Thornberg U, Frostell A, et al. A randomized trial of continuous versus intermittent skin-to-skin contact after premature birth and the effects on mother-infant interaction. Adv Neonatal Care. 2020;20(3)

51. Mäntymaa M, Puura K, Luoma I, et al. Infants' social withdrawal and parents' mental health. Infant Behav Dev. 2008;31(4):606-613.

52. Trevarthen C, Aitken KJ. Infant intersubjectivity: research, theory, and clinical applications. J Child Psychol Psychiatry. 2001;42(1):3-48.

53. Menahem S. Conservation-withdrawal reaction in infancy? An underdescribed entity. Child Care Health Dev. 1994;20(1):15-26.

54. Smith-Nielsen J, Lønfeldt N, Guedeney A, et al. Implementation of the Alarm Distress Baby Scale as a universal screening instrument in primary care: feasibility, acceptability, and predictors of professionals' adherence to guidelines. Int J Nurs Stud. 2018;79:104-113.

55. Guedeney A, Fermanian J. A validity and reliability study of assessment and screening for sustained withdrawal reaction in infancy: the Alarm Distress Baby scale. Infant Ment Health J. 2001;22(5):559-575.

56. Carla PM, Menahem S, Viaux S, et al. Alarm Distress Baby Scale (ADBB) in assessing social withdrawal in infants with an underlying organic illness - congenital heart disease, prader willi syndrome, cleft lip and/or palate - an overview. IMR. 2020;6.

57. Seidman G, Unnikrishnan S, Kenny E, et al. Barriers and enablers of kangaroo mother care practice: a systematic review. PLOS ONE. 2015;10(5):e0125643 